\title{
Emergency gate vibration of the pipe-turbine model
}

\author{
Andrej Predin ${ }^{\mathrm{a}, *}$ and Roman Klasinc ${ }^{\mathrm{b}}$ \\ ${ }^{a}$ Faculty of Mechanical Engineering, University of \\ Maribor, Smetanova 17, SI-2000 Maribor, Slovenia \\ E-mail: andrej.predin@uni-mb.si \\ ${ }^{\mathrm{b}}$ Department of Hydraulic Structures and Water \\ Resources Management, Technical University Graz, \\ Stremayrgasse 10/II, A-8010 Graz, Austria \\ E-mail:klasinc@kwb.tu-graz.ac.at
}

Received 14 October 1998

Revised 23 November 1999

The vibration behavior of an emergency gate situated on a horizontal-shaft Kaplan turbine is studied. The analysis and transfer of the dynamic movements of the gate are quite complex. In particular the behavior is examined of the emergency gate for the case when the power unit is disconnected from the system or there is a breakdown of the guide vane system at the moment when the maximal head and capacity are achieved. Experimental-numerical methods both in the time domain and in the frequency domain are employed. Natural vibrations characterize a first zone, corresponding to relatively small gate openings. As the gate opening increases, the vibration behavior of the gate becomes increasingly dependent on the swirl pulsations in the draft tube of the turbine. Finally, the data transfer from the model to the prototype by use of the dynamic similitude law is discussed.

\section{Introduction}

Scale model studies were performed to determine the forces acting vertically on an emergency gate and its suspension. The only way of studying the behavior of the emergency gate's vibrations under extreme flow conditions was to use a physical scale model. Results were already available from several studies relating to flow under flat gates and flow induced vibrations of solid structures $[2,7,8,10]$. In the case under study, however, the flow contained an additional swirl com-

\footnotetext{
*Corresponding author.
}

ponent, which was generated by the turbine. A pronounced swirl component was produced when the turbine was running at an increased speed (disconnection from the system). Likewise, lock-up of the guide vane system risked a situation which could lead to extreme flow conditions. The forces acting on the emergency gate and its suspension contained both static and dynamic components.

After computing these forces, we were faced with the problem of transferring the model conditions to the prototype. For the static forces, transfer to the prototype is possible by using Froude's laws of similitude. The section under study was short enough to allow the frictional forces to be ignores which is a prerequisite for using Froude's laws. The static forces resulting from the swirling flow can be transferred in the same way. The transfer mechanisms of the dynamic forces, however, can only be dealt with by using the dynamic laws of similitude. Different vibration mechanisms were found to develop as a function of the opening size of the emergency gate. Characteristic vibrations were seen to occur for the small gate openings. As the gate was lifted, vibrations generated by the pulsations of the turbine swirl became increasingly important. The goal of this study was to establish the vibration behavior of the gate for different openings.

\section{Model set-up and measuring equipment}

A transparent material (Plexiglas) was used to form the model, while the turbine blade, the guide vane system, and the gate leaf were fabricated from brass (Fig. 1), which made it possible to study the cavitation swirl formed under certain flow conditions. Figure 1 shows the suspension of the gate very clearly. In Fig. 2.a, longitudinal section through the power unit is shown. The kinematics of the emergency gate were registered by means of a force and an acceleration transducer. Figure 2 also shows the arrangement of the measuring transducers. We generally used transducers allowing the non-distorted registration of signals to fre- 


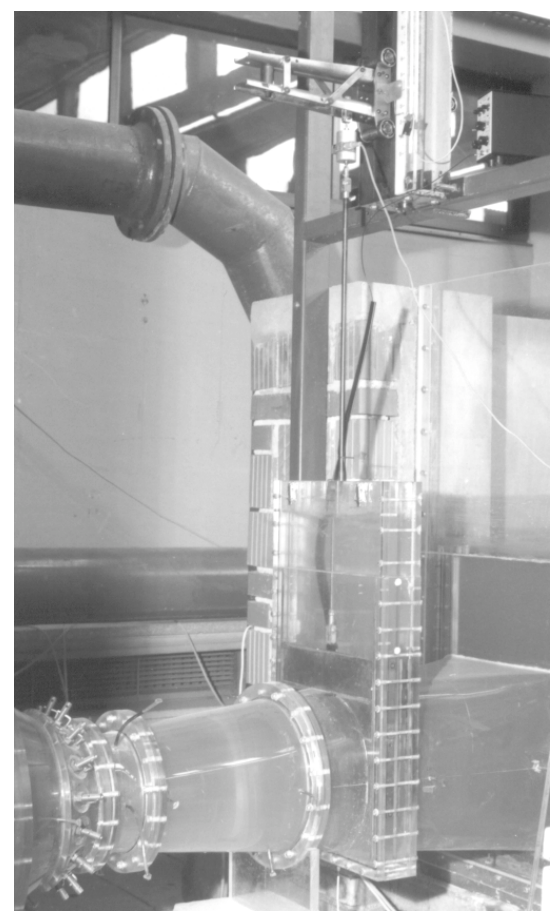

Fig. 1. Kaplan turbine model - bulb unit.

quencies of at least $300 \mathrm{~Hz}$. For the efficient acquisition and analysis of the measured data we used an A/D computer board (Burr Brown with Visual Designer software). This set-up had a scanning rate of $2 \mathrm{kHz}$ per channel. The blocks of 8192 measured digits were used for preparing the power spectra and the correlation analyses.

The water flow over the top of the gate shows up as an additional problem at the position where the acceleration transducer was placed; so, the accelerometer was covered with the plastic foil pipe to keep the electrical contacts dry.

The dynamic response of the measurement system was determined before each measurement; meaning, the frequency and the system damping of gate's instrumentation system must be determined. The natural oscillation of the gate, which is supported by the spindle, was excited by a force pulse. The strong damping of the natural oscillation of the gate is evident (Fig. 3) from the relatively large decrease on the magnitude of the oscillation. The system damping is determined by logarithm decrement of two successive gate oscillation magnitudes using the equation

$$
\delta=\ln \left(\frac{M_{i}}{M_{i+1}}\right)
$$

For the system damping, if it is operated in air, the damping ratio is 0.172 , and if it is operated in water the ratio is 0.195 . The natural frequencies are determined using FFT analyses of the oscillation data for the two cases. First, for the case when the gate was surrounded by air, and second, when the gate was surrounded by water. In first case, the dominant natural oscillation frequency of the gate is determined to be between 310 and 320 Hertz, and in the second case it is between 30 and 36 Hertz (Fig. 4). For the both cases, the frequency peak is quite clearly evident. In both power spectra records, some other spectral frequencies are present, but their magnitudes are much smaller. Force and pressure transducers were calibrated for each trial. The force transducer was calibrated by weights and the pressure transducer was calibrated using a known pressure. Linear responses were achieved at both cases.

\section{Measurement results}

Measurements were performed at the model's maximal head difference (difference between upper and lower water level) at different gate openings. The flow rate, turbine runner speed, pressure at the draft tube below the gate, acting force, and acceleration at the gate in the vertical direction were measured. From force-time history records (Fig. 5) different force vibrations at different gate opening were evident. At the gate openings of up to $16.7 \%$ the force vibration with higher frequency is evident. When the gate opening is increased, the force vibration frequency decreased. The average value of the acting force increases by enlarging the gate openings up to $83.3 \%$, and then it decreases after that. The reason for that situation is that at the higher flow rates (larger gate openings) the swirl flow occurs at the draft tube. The extreme swirl flow is present at the cavitating turbine operating conditions (at maximal flow rates). With this situation, the spiral vortex rope (or vortex core) appears in a draft tube and often causes violent pressure fluctuation, which is called the draft tube surge. That vortex core is identified by pressure measurements and it is already present at approximately a $28 \%$ gate opening. The vortex core appears quickly in the flow in the draft tube, when the turbine model is operated at extreme operating conditions. At normal turbine operating regimes, the swirled flow is present at the draft tube, but not in such an extreme cavitating form. Pressure-time history records (Fig. 6) confirm the existing vortex core in the flow at the draft tube; the pulsating pressure form is evident at the larger gate openings. The pressure frequency increases from $3.2 \mathrm{~Hz}$ at a $28 \%$ gate opening to $4.64 \mathrm{~Hz}$ 


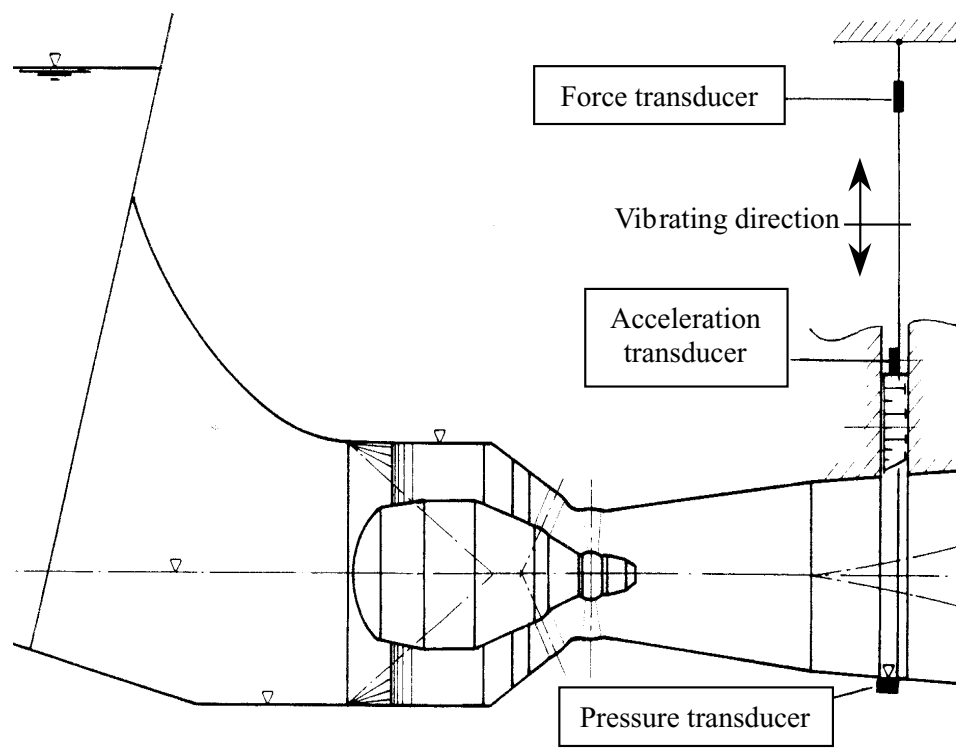

Fig. 2. Transducer arrangement at tested model.

a)

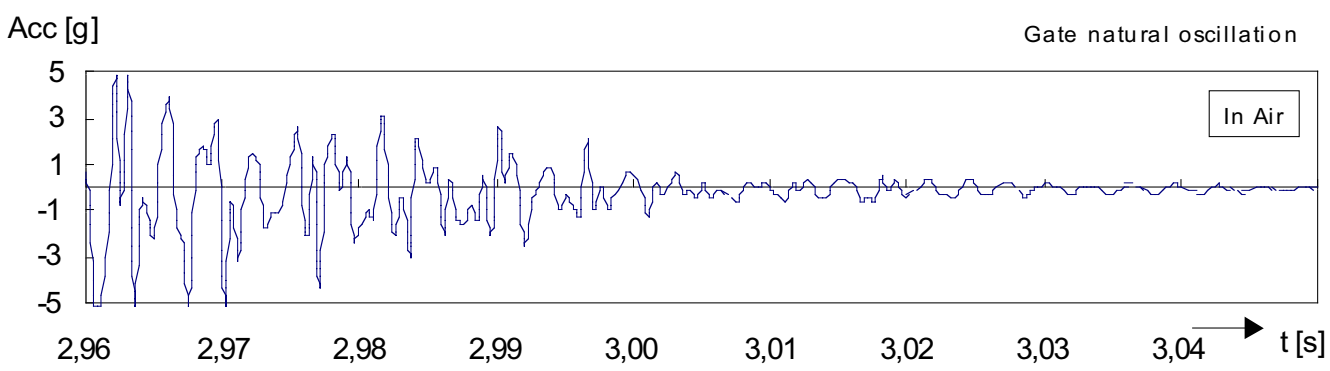

Acc [g]

Gate natural oscillation

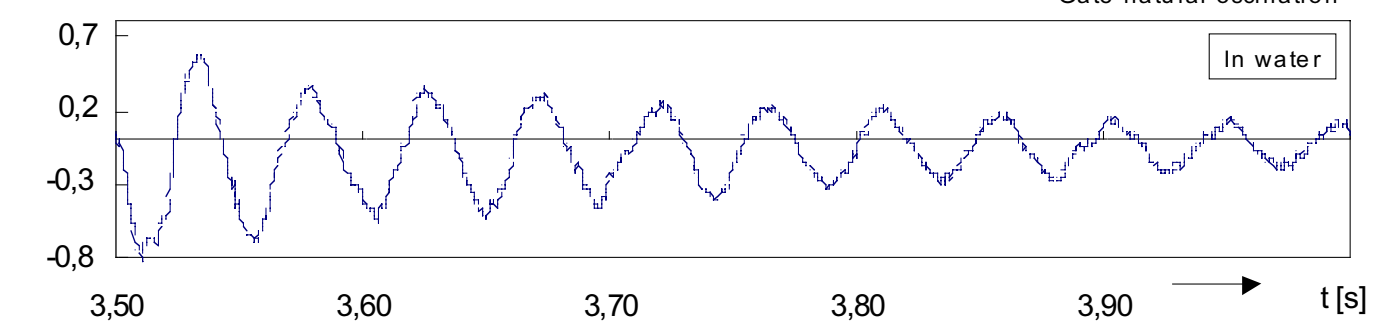

Fig. 3. Time history records of the gate natural oscillation in air (a) and in water (b).

at on approximately $83 \%$ gate opening and than decreases to $3.66 \mathrm{~Hz}$ at an approximately $94 \%$ gate opening. The reason for this frequency change is probably the fact that in the vortex core there is more and more air/water-exhalation, and the vortex friction increases. Two dominant force-frequency peak areas are shown in the frequency "waterfall diagram" (Fig. 7) and indicate a dependency of the amount of the gate opening. Dominant frequency peaks are located at two areas between 27 and $54 \mathrm{~Hz}$ and between 297 and $324 \mathrm{~Hz}$. These two areas approximately correspond to the natural frequencies. At larger gate openings, the higher spectral frequencies are present in the power spectra record. These frequencies probably correspond to the flow turbulence at higher flow rates a cross the draft tube. It can be shown that the gate vibrates with natural frequencies at the smaller gate openings and with the frequency that correspond to the vortex rope round-up 


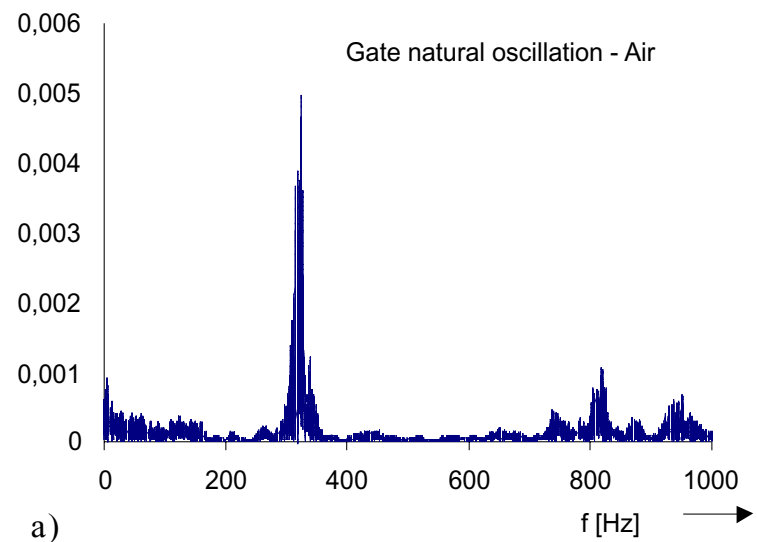

Fig. 4. Power spectra records of the gate natural vibration, determined in air (a) and in water (b).

frequencies at higher flow rates or at larger gate openings. This fact is also confirmed by correlation analyses between pressure and force, acceleration and force, and between the acceleration and pressure. Figure 8 shows the correlation between force and flow pressure; a large correlation between the measured data of force and pressure. At smaller gate openings the observable correlation between force and pressures is not evident in the correlation record.

Below a $20 \%$ gate opening the small values of the acting force are evident. The gate vibrations at this area (A, Fig. 9) had a frequency near to the gate's natural frequencies. In this operating area, the gate vibrations are self excited gate vibrations. The average values of the acting force increase (Fig. 9) when the gate is open up to $20 \%$. In this area, the alternating part of the force is relatively small. The force frequency is higher than the corresponding natural frequency, so it can be concluded that this is the beginning of the transition area of self excited gate vibrations and forced vibrations. Gate openings up to $28 \%$ are in a transitional area where gate vibrations are both self excited and force excited by the vortex rope. In the area designated by (B) in Fig. 9, the alternating part of the acting force increases further. In area (C), with up to $70 \%$ gate opening, the vortex rope is fully developed. For this area, an almost constant acting average force and constant alternating force magnitude are characteristic. Moreover, in area (C) the force vibrating frequency is also constant between 3.17 and $4.6 \mathrm{~Hz}$. In the area of gate opening between $60 \%$ and $83 \%$ the frequency is almost constant, approximately $4.5 \mathrm{~Hz}$. On that basis, one can conclude that the flow vortex frequency is almost constant. By further increasing the gate opening from $70 \%$ and up to $95 \%$, the average value of the act-

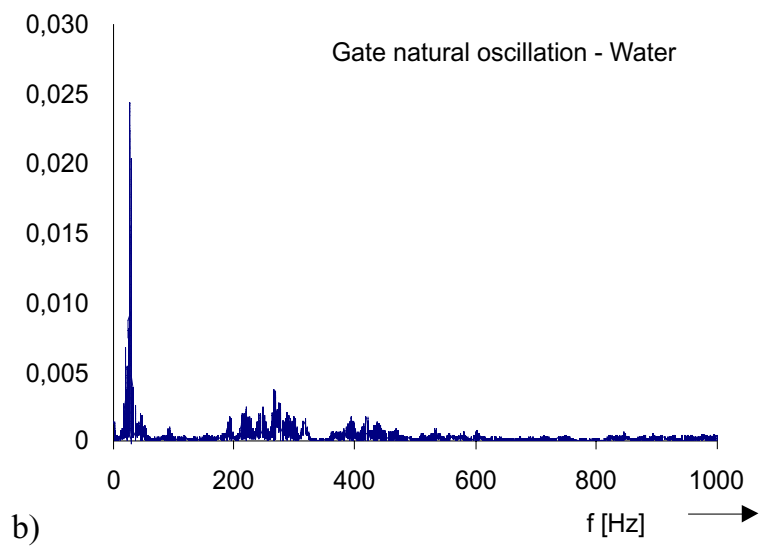

ing force decreases, but at the same time the alternating part of the force remains relatively large and does not decrease until a gate opening of almost $100 \%$. The force pulsating frequency in the area (D) decreases. It can be concluded that the vortex rope filled up with the water steam, which is characteristic for the cavitating operating regime. The vortex rope rolled-up frequency decrease could be caused by the increase of the vortex friction in the flow in the draft tube.

\section{Gate vibration mathematical model}

As the measured results show, the gate vibrations could be divided into the three types: self excited vibrations; forced vibrations, caused by pressure oscillations in the draft tube [1] and at the combination of those two.

The self-excited gate vibrations can be expressed as one a degree-of-freedom system with the viscous damping vibration equation

$$
a_{F}=m \ddot{y}+\xi \dot{y}+k y
$$

where $y$ is the displacement due to the vibration and its time derivations, $k$ is the "spring" rigidity, $\xi$ is damping coefficient, $m$ is the gate mass with the spindle and acceleration transducer mass, and $a_{F}$ is the alternating part of the hydraulics force acting on the gate in the vertical direction and is determined by

$$
a_{f}=F_{h}-F_{h, \text { mean }}
$$

In Eq. (3), $F_{h}$ is the common hydraulics acting force and $F_{h, \text { mean }}$ is the mean value or mean hydraulics force that is acting on the gate lip in the vertical direction. All needed parameters from equation (2) could be de- 

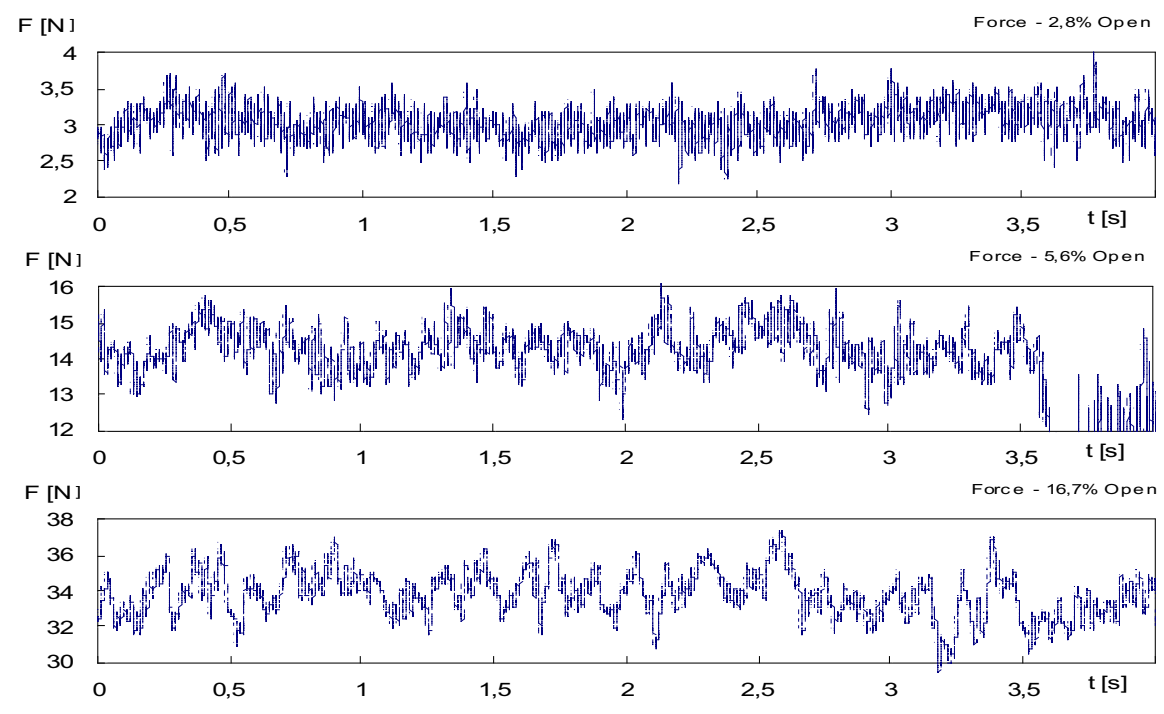

$\mathrm{F}[\mathrm{N}] \quad$ Force $-27,8 \%$ Open

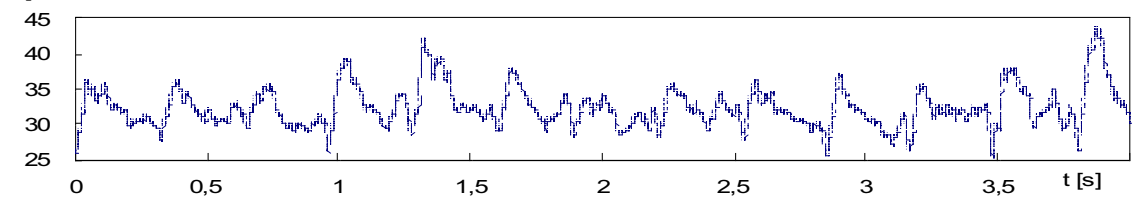

$\mathrm{F}[\mathrm{N}] \quad$ Force $-38,9 \%$ Open
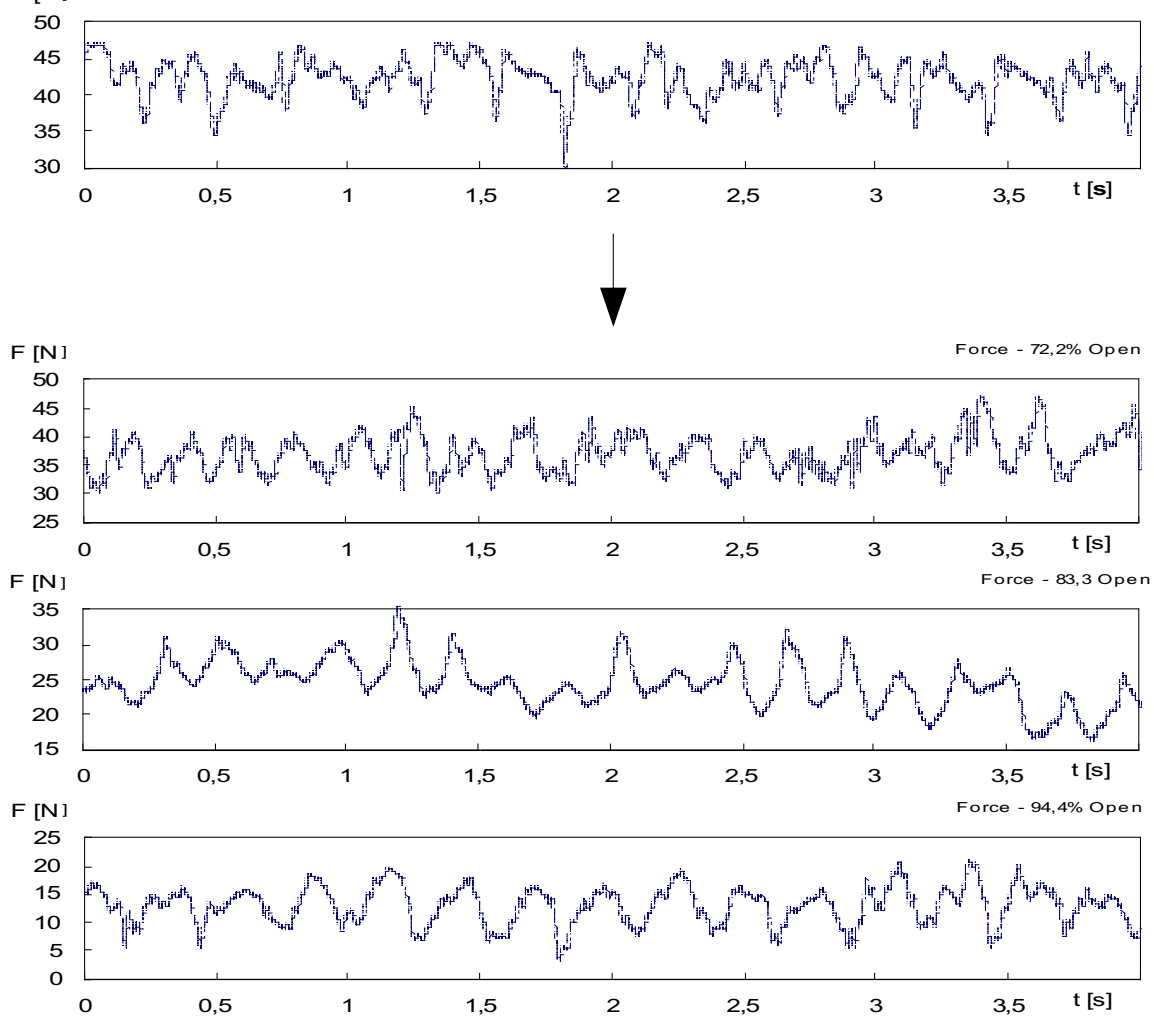

Fig. 5. Time history records of acting force measured at the gate suspension in vertical direction at different gate openings, from $2.8 \%$ up to $94.4 \%$. 


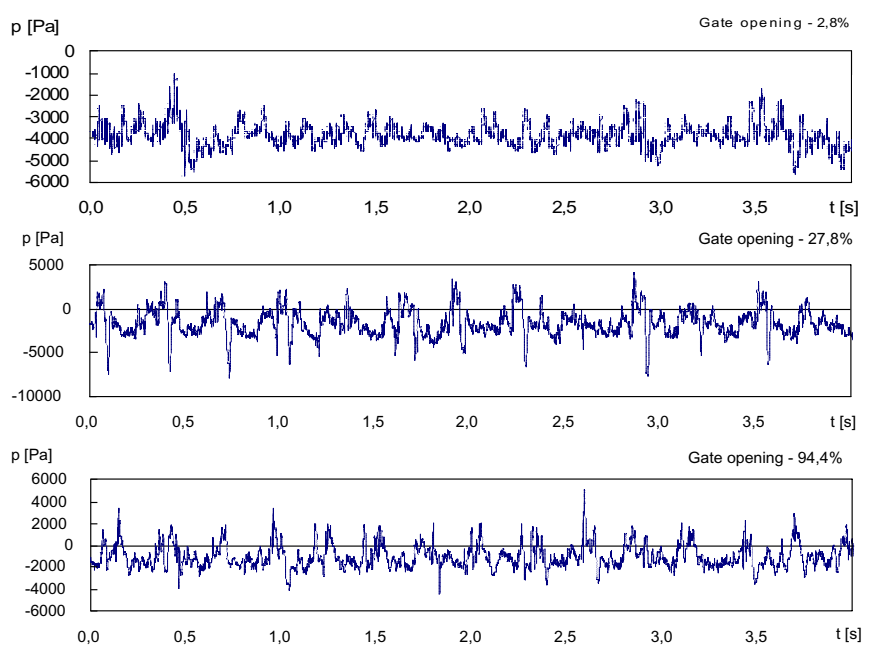

Fig. 6. Time history records of the flow pressures at different gate openings.

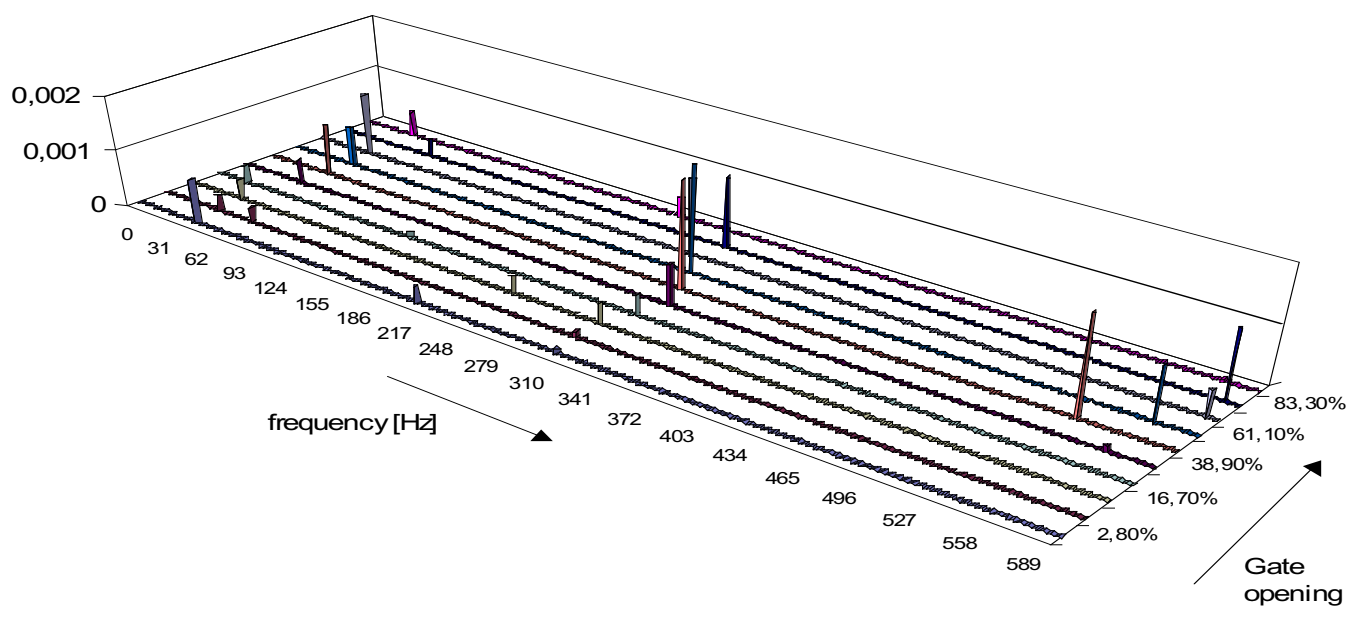

Fig. 7. "Waterfall" power spectra record of acting force at different gate openings.

termined theoretically $(m, k)$ or experimentally $\left(a_{F}, \xi\right)$ by well-known procedures. The unknown values of the alternating part of the hydraulics force $a_{F}$ and the damping coefficient $\xi$ could be theoretically determined using equations [8], as can the vertical gate vibration. The alternating hydraulics force is

$$
a_{F}=c_{F} \rho g B \Delta H^{\prime}
$$

where $c_{F}$ is the force coefficient, $\rho$ is flow density, $g$ is acceleration due to gravity, $B$ is the gate lip area, and $\Delta H^{\prime}$ is the acceleration of the water column.

$$
\Delta H^{\prime}=\frac{-L}{g A_{D}} \dot{Q},
$$

where $L$ is the culvert or diffuser length, $A_{D}$ is the diffuser's cross-sectional area, and $\dot{Q}$ is the time derivation of the discharge equation.

$$
Q=A_{\text {eff }} \sqrt{2 g \Delta H},
$$

where $\Delta H$ is the local head difference across the gap and $A_{e f f}$ is the effective gape area.

$$
A_{e f f}=\varphi \delta w,
$$

where $\varphi$ is the flow loss coefficient $(0.9-0.8), \delta$ is the temporary gate opening and $w$ is the gate width.

When gate vibrations are excited by close to a singlefrequency harmonic excitation caused by vortex rope at the draft tube, or by a pressure disturbance in the draft tube [3], (at area B, C and D, Fig. 9). Governing equations $[5,11]$ for motion of viscously damped in the general form 

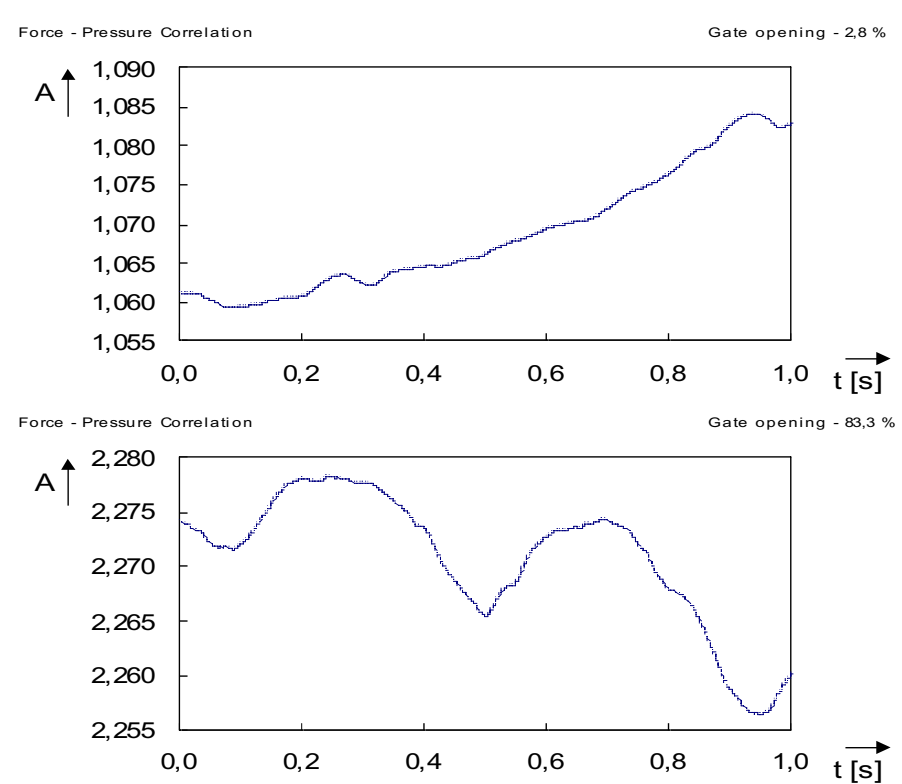

Fig. 8. Correlation record between acting force and flow pressures in the draft tube.

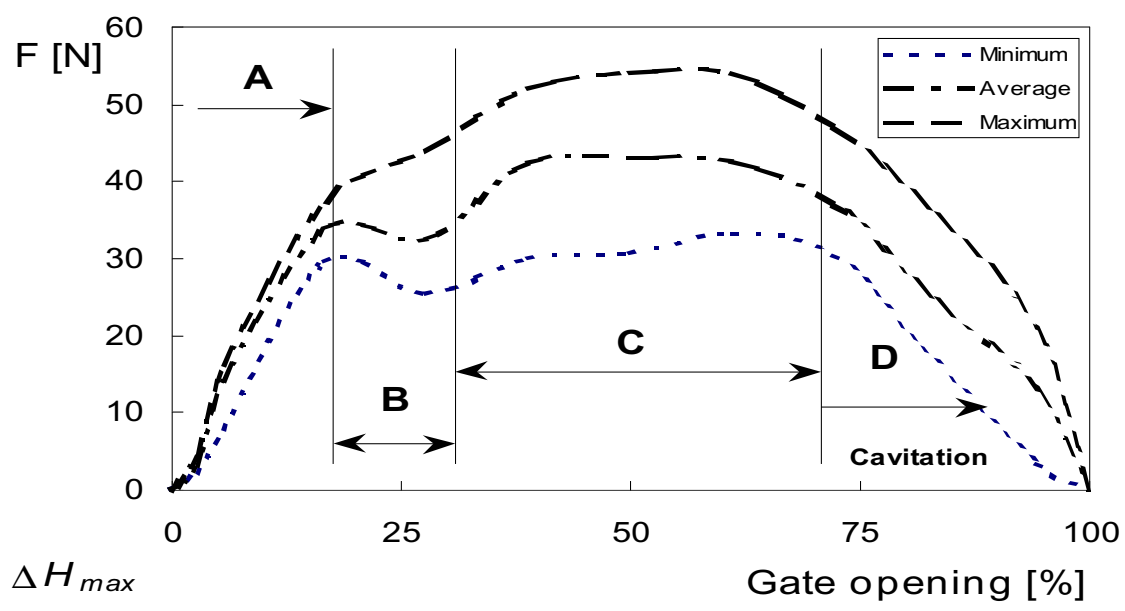

Fig. 9. Maximal, minimal and average values of the acting force in dependency of gate opening.

$$
\ddot{y}+2 \xi \omega_{n} \dot{y}+\omega^{2} y=\frac{F}{\tilde{m}} \sin \left(\omega_{\text {rope }} t+\psi\right)
$$

where $\omega_{n}$ is the natural frequency, $\xi$ is the damping ratio of natural oscillation, $F$ is the excited force, and $\tilde{m}$ is the added mass considering the gate vibration in the water, $\omega_{\text {rope }}$ is the frequency of the vortex rope, and $\psi$ is the phase difference between the excitation and the closely sinusoidal excitation. The vortex rope rolled-up frequency can be modeled using as a base formulation from [9], which is valid for the Francis turbine. The new, modified formulation is adopted for the Kaplan turbine and it takes the form

$$
\omega_{\text {rope }}=\frac{2 \Gamma}{D_{h} e^{\left(n / n_{\max }\right)^{0,4}}},
$$

where $D_{h}$ is the turbine runner hub diameter, $n$ is the runner speed, $n_{\max }$ is the runaway runner speed, and $\Gamma$ is the circulation that can be determined as the sum of the circulation in the axial direction $\Gamma_{a x}$ and, the circulation $\Gamma_{p}$ around the runner blade profile

$$
\Gamma=\Gamma_{a x}+\Gamma_{p}
$$

The circulation in the axial direction (axial direction of the draft tube) is determined as

$$
\Gamma_{a x}=\frac{\pi^{2} n}{z}\left(D_{t}^{2}-D_{h}^{2}\right)
$$




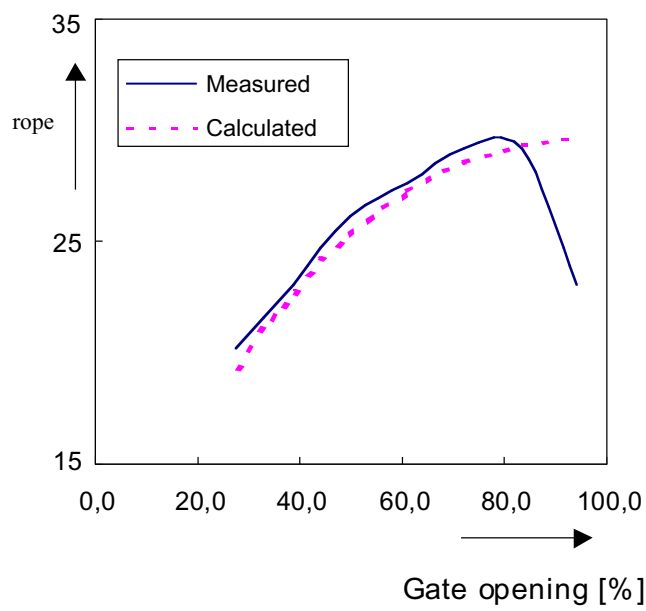

Fig. 10. Vortex rope frequency.

where $z$ is the runner blade number and $D_{t}$ is the runner (tip) diameter. The circulation around the runner blade profile, considered Euler's main equation for axial turbomachines, is determined as

$$
\Gamma_{p}=g \Delta H_{t o t} \frac{\pi D_{h}}{z}
$$

where $\Delta H_{t o t}$ is the total head across the turbine runner.

Equation (10) results are in good agreement with the measured data for the vortex rope frequency (Fig. 10). For higher gate openings there is a larger disagreement between the calculated and measured vortex rope frequency. This is caused by the fact that the measured frequency dropped rapidly while the vortex rope was filling up with water vapor and collapsing. With the substitution of Eq. (10) in to (9), good results for the calculated gate vibrations are achieved. As an example, Fig. 11 shows the calculated results for force vibrations at the gate in the vertical direction for a gate opening of $83.4 \%$ as compared to measured results. The good agreement of the vibration frequencies is evident, whereas the vibration magnitudes have less agreement. The reason the calculated and measured vibration magnitudes not agree could be because the other spectral frequencies such as the runner speed frequency and the runner blade frequency and their higher harmonics are not considered.

\section{Parameter transfer from model to prototype}

The static behavior of the gate could be transferred from model to the prototype conditions using Froude's law of similitude. The oscillating pressure distribution, to the gate in the vertical direction increases the oscillating force acting normal to the gate lip. The right side of Eq. (9), gives the force, where $\omega_{\text {rope }}$ is the frequency of vortex rope. All parameters on the right side of Eq. (9) depend on the fluid properties and the geometry of the gate or gate lip. For force and frequency transfer we can use the general equation [12],

$$
F=f(V, \rho, \mu, L)
$$

where $V$ is flow velocity, $\rho$ is flow density, $\mu$ is fluid viscosity, and $L$ is the gate lip area minus the length. Using the Buckingham $\pi$ theorems in equation (14) allows the equation to be rewritten as a relationship between dimensionless parameters, such as Reynolds

$$
R e_{m}=\frac{\rho_{m} V_{m} L_{m}}{\mu_{m}}=\frac{\rho_{p} V_{p} L_{p}}{\mu_{p}}=R e_{p}
$$

and Froude

$$
F r_{m}=\frac{V_{m}^{2}}{g L_{m}}=\frac{V_{p}^{2}}{g L_{p}}=F r_{p}
$$

numbers. Using these dimensionless parameters, the transfer between the model and prototype conditions can be established. Considering the dynamic similarities that exist when the model and the prototype have the same length-scale ratio, time-scale ratio, and forcescale (or mass-scale) ratio, shows that the dynamic similarity exists simultaneously with the kinematic similarity, if the model and the prototype force and pressure coefficients are identical. This is certain where there is free surface flow and when the model and the prototype have the same Reynolds, Froude, Weber, and Euler (cavitation) numbers.

The Froude number contains only length and time dimensions, and hence is a purely kinematic parameter which fixes the relation between length and time [6]. So, from equation (16), if the length scale is

$$
L_{m}=\lambda L_{p},
$$

where $\lambda$ is a dimensionless ratio, the velocity scale is

$$
\frac{V_{m}}{V_{p}}=\left(\frac{L_{m}}{L_{p}}\right)^{1 / 2}=\sqrt{\lambda}
$$

The time scale is

$$
\frac{T_{m}}{T_{p}}=\frac{L_{m} / V_{m}}{L_{p} / V_{p}}=\sqrt{\lambda}
$$

or the frequency scale is

$$
\frac{\omega_{m}}{\omega_{p}}=\frac{V_{m} / L_{m}}{V_{p} / L_{p}}=\lambda^{-1 / 2}
$$




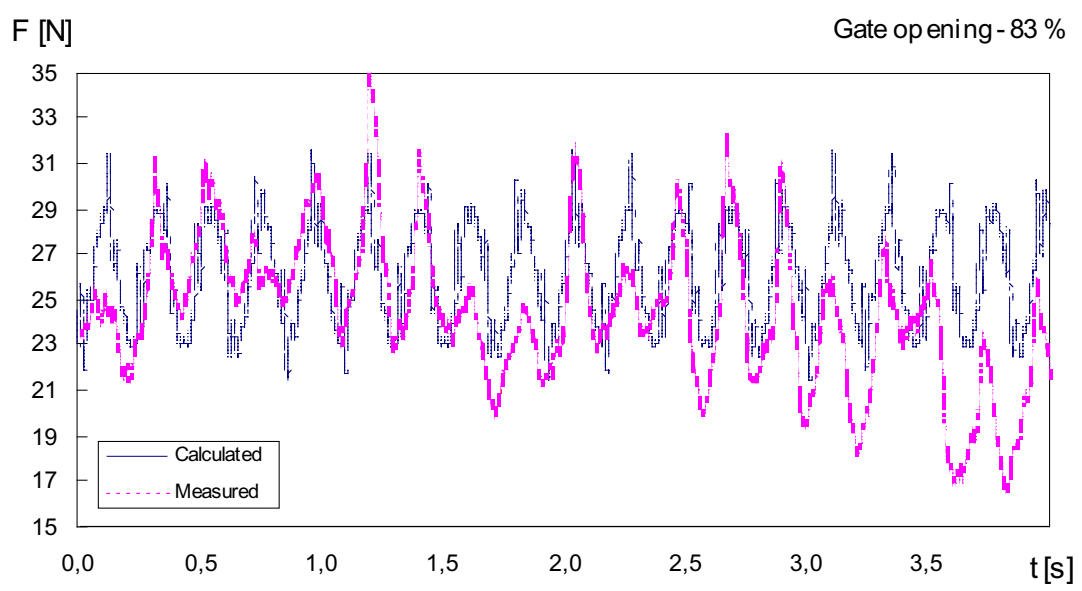

Fig. 11. Calculated and measured force comparison.

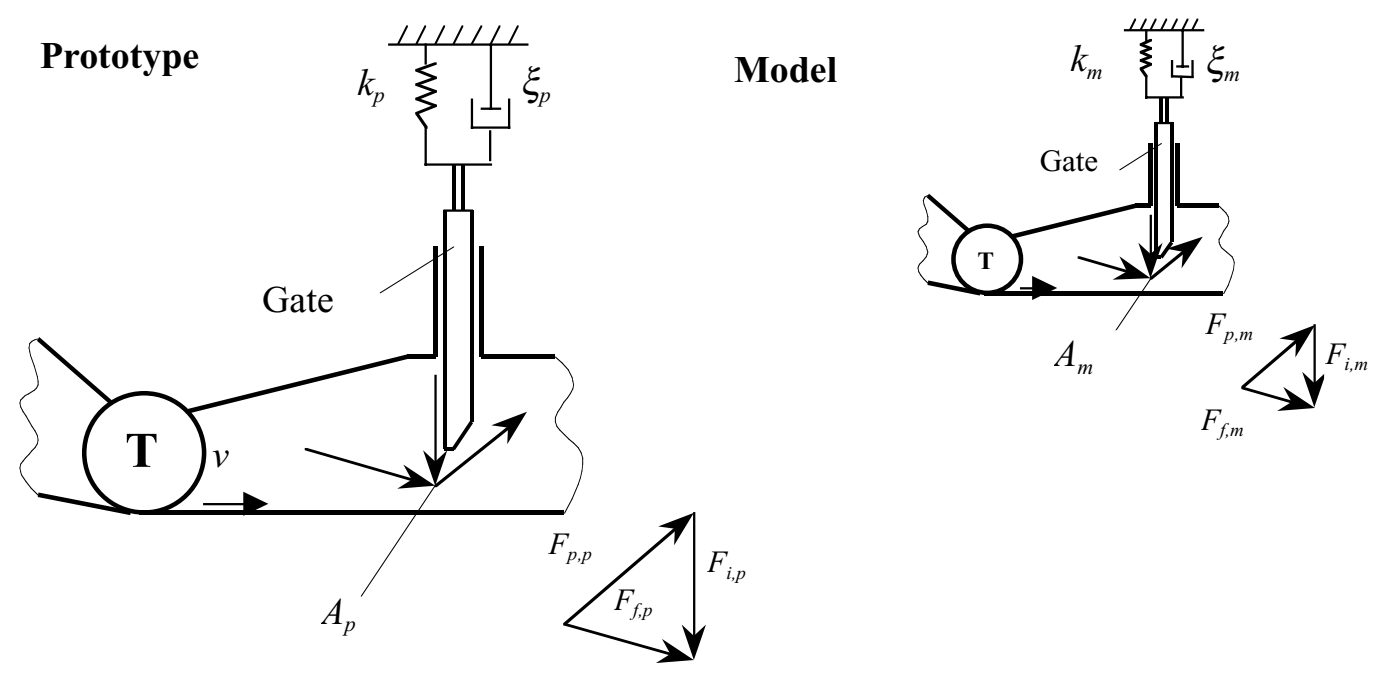

Fig. 12. Gate - model and prototype and kinematically similar flows.

using these equation the vortex rope frequency from the model to the prototype can be predicted. The excitation force, at the right hand side of equation (9), can be predicted from the model to the prototype considering Newton's law, which says that the inertial force is the sum of the pressure, gravity, and the friction force. Thus, the dynamic similarity laws listed above ensure that each of these forces will be in the same ratio and have equivalent directions between the model and the prototype. Let us examine two dynamically similar, incompressible, viscous flows through the gates, a model flow and a prototype flow (Fig. 12). Neglecting the body forces, two types of forces acting on each flow particle may be distinguished, namely, shear force and pressure force. If the inertia term in Newton's law is now written as a D'Alembert force, ma, we may consider the sum of the two external forces plus the D'Alembert force as equal to zero and, thus, in equilibrium. The triangle of forces could be established for each point of the flow as is shown in Fig. 12 for points $A_{m}$ and $A_{p}$. According to the rules of dynamic similarity, the triangles of forces for these points are similar, since the sides of the triangles must be parallel. We may now form the following equations, which apply to all corresponding points:

$$
\begin{aligned}
\frac{(\text { Press., } \mathrm{F})_{m}}{(\text { Press., } \mathrm{F})_{p}} & =\frac{(\text { Frict., } \mathrm{F})_{m}}{(\text { Frict., } \mathrm{F})_{p}} \\
& =\frac{(\text { Inert., } \mathrm{F})_{m}}{(\text { Inert., } \mathrm{F})_{p}}=\text { const. }
\end{aligned}
$$

The following relations may be derived from these equations: 


$$
\begin{aligned}
& \frac{(\text { Inert., } \mathrm{F})_{m}}{(\text { Frict., } \mathrm{F})_{m}}=\frac{(\text { Inert., } \mathrm{F})_{p}}{(\text { Frict., } \mathrm{F})_{p}}=(\text { const. })_{1}, \\
& \frac{(\text { Inert., } \mathrm{F})_{m}}{(\text { Press., } \mathrm{F})_{m}}=\frac{(\text { Inert., } \mathrm{F})_{p}}{(\text { Press., } \mathrm{F})_{p}}=(\text { const. })_{2},
\end{aligned}
$$

Using derivations [4], the end equation (20) can be written as

$$
\left(\frac{\Delta p}{\rho V^{2}}\right)_{m}=\left(\frac{\Delta p}{\rho V^{2}}\right)_{p}
$$

where $\Delta p$ is the difference between the static pressure at the draft tube entrance and the static pressure under the gate, and $V$ is the entrance flow velocity at the draft tube entrance. From Eq. (23), it may be concluded that a necessary condition for dynamic similarity for the particular flows under study is the equality of the Reynolds number and the Euler number between the flows, using the same flow parameters and geometrical scale. In our case, we can replace $\Delta p$ in equation (23) with $F /(B w)$, which is proportional to $\Delta p$ (B is the gate lip area and $w$ is the gate width). Using this substitution, we can write

$$
\frac{F_{m} /\left(B_{m} w_{m}\right.}{\rho_{m} V_{m}^{2}}=\frac{F_{p} /\left(B_{p} w_{p}\right.}{\rho_{p} V_{p}^{2}}
$$

hence the force on the prototype is

$$
F_{p}=F_{m} \frac{\rho_{p} V_{p}^{2}}{\rho_{m} V_{m}^{2}}\left(\frac{B_{p} w_{p}}{B_{m} w_{m}}\right) .
$$

Assuming a ratio $\lambda$, from geometrical similarity, equation (17), between the model and the prototype, equation (26) can be rewritten as

$$
F_{p}=F_{m} \frac{\rho_{p}}{\rho_{m}} \lambda^{3}
$$

which applies to the incompressible flow, where $\rho_{p}=$ $\rho_{m}=\rho=$ constant, i.e., Froude's law.

The gate guiding system rigidity or the "spring" rigidity $k$ of the model and the prototype and the gate mass, respectively, could be in the same geometric ratio

$$
\frac{k_{p}}{k_{m}}=\frac{m_{p}}{m_{m}}=\lambda \text {. }
$$

If so, then it is acceptable that the damping coefficients are also the in the same ratio

$$
\frac{\xi_{p}}{\xi_{m}}=\lambda \text {. }
$$

If the stiffness, mass, and damping coefficients do not have the same ratio, then we must measure or calculate them directly from the prototype. Allowing for the above equations (26) and (29), the force vibration at the prototype emergency gate can be predicted from calculations using equation (9).

\section{Conclusions}

The emergency gate vibrations that occur by closing the draft tube of the Kaplan horizontal shaft turbine are generated as flow-excited vibrations. In the area of small gate openings, about up to $20 \%$, the vibrations are self excited. The common vibrating frequency is close to the gate's natural frequency which means that the gate vibrates in the vertical direction with its natural frequency. Fortunately, the magnitude of these vibrations is small, up to $20 \%$ of hydrodynamic force that of the. With larger gate openings, from $25 \%$ to $70 \%$, a vortex flow in the draft tube is created. Vortex excited gate vibrations are generated simultaneously which are almost sinusoidal. The common vibration frequency is equal to the flow pressure pulsation frequency. The pressure pulsation in the draft tube is a consequence of the vortex rope created in the flow. The magnitude of the pulsation is up to $50 \%$ of the average hydraulics force at these openings. With maximal gate openings (from $75 \%$ to $100 \%$ ) the vibration magnitudes increase intensively and are up to $70 \%$ of the average hydrodynamics force at these openings. However, the average value of the hydraulics force at these openings decreases to very small values when the gate is fully open.

Finally, it can be concluded that the critical moment occurs when the extreme gate loading exists, that is, at the first quarter of the gate closing from $100 \%$ to $75 \%$ of the gate opening when the maximal flow rate in the draft tube is achieved. In this operating area the cavitating operating conditions at the turbine runner and in the turbine draft tube show up. That means that the vortex from the turbine runner is changed into a cavitating vortex rope that is rolled-up at the draft tube.

Transferring parameters from the model to the prototype is possible by allowing for dynamic similarity, the Froude law, and geometrical similarity. On that basis, it is possible to predict force vibrations at the prototype.

\section{Acknowledgments}

The authors thank the Institute of Water Buildings and Water Sciences at Graz Technology University in Austria for their support of these investigations as well as for permission to publish the results. 


\section{References}

[1] G. Angelico, A. Di Monaco, P. Fanelli and P. Molinaro, Transfer matrix of a mechanical device for damping pressure oscillations in a hydroelectric power plant, Proceedings of the 6th IAHR Meeting WG1, Lausanne, 1993.

[2] D.R. Blevins, Flow-Induced Vibration, second edition, Van Nostrand Reinhold, New York, 1990.

[3] H. Brekke, Ongoing and planned work at the Norwegian Institute of Technology, related to hydraulic machinery under steady oscillatory conditions, 7th IAHA meeting, WG1, Ljubljana 1995.

[4] V.R. Giles, J.B. Evett and C. Liu, Schaum's outline of: Theory and problems of Fluid mechanics and hydraulics, McGrawHill Book Company, 1994.

[5] P.J. Holman and R.J. Lloyd, Fundamentals of Mechanical Vibrations, McGraw-Hill Book Company, 1993.

[6] T. Jacob, Similitudes in stability of operation test for Francis turbines, Hydropower \& Dams, Jan 1994.

[7] R. Klasinc and A. Predin, Vibrations of an Emergency Gate in the Draft Tube of a Horizontal - Shaft Turbine, Proceedings of the 28th IAHR congress, Graz, 1999.

[8] P.A. Kolkman, Flow-induced gate vibrations, thesis work, 1976, Delft Hydraulics Laboratory, publication no. 164.

[9] M. Nishi, X. Wang and T. Takahashi, A short Note on the Rotating Frequency of Vortex Rope in a Draft Tube, 7th IAHR Meeting, WG1, Ljubljana 1995.

[10] K. Ogihara, Y. Ueda, I. Minagawa and T. Ueda, Fields test of vibration of draft gate in pump storage station, Proceedings of Modeling, Testing and Monitoring for Hydropower Plants-III Conference, Aixen-Provence, France, October 1998.

[11] A. Predin, Torsional Vibrations at Guide-Vane Shaft of PumpTurbine Model, Shock \& Vibration, Volume 4, Issue 3, 1997, $153-162$.

[12] M.F. White, Fluid Mechanics, second edition, McGraw-Hill Book Company, 1986. 

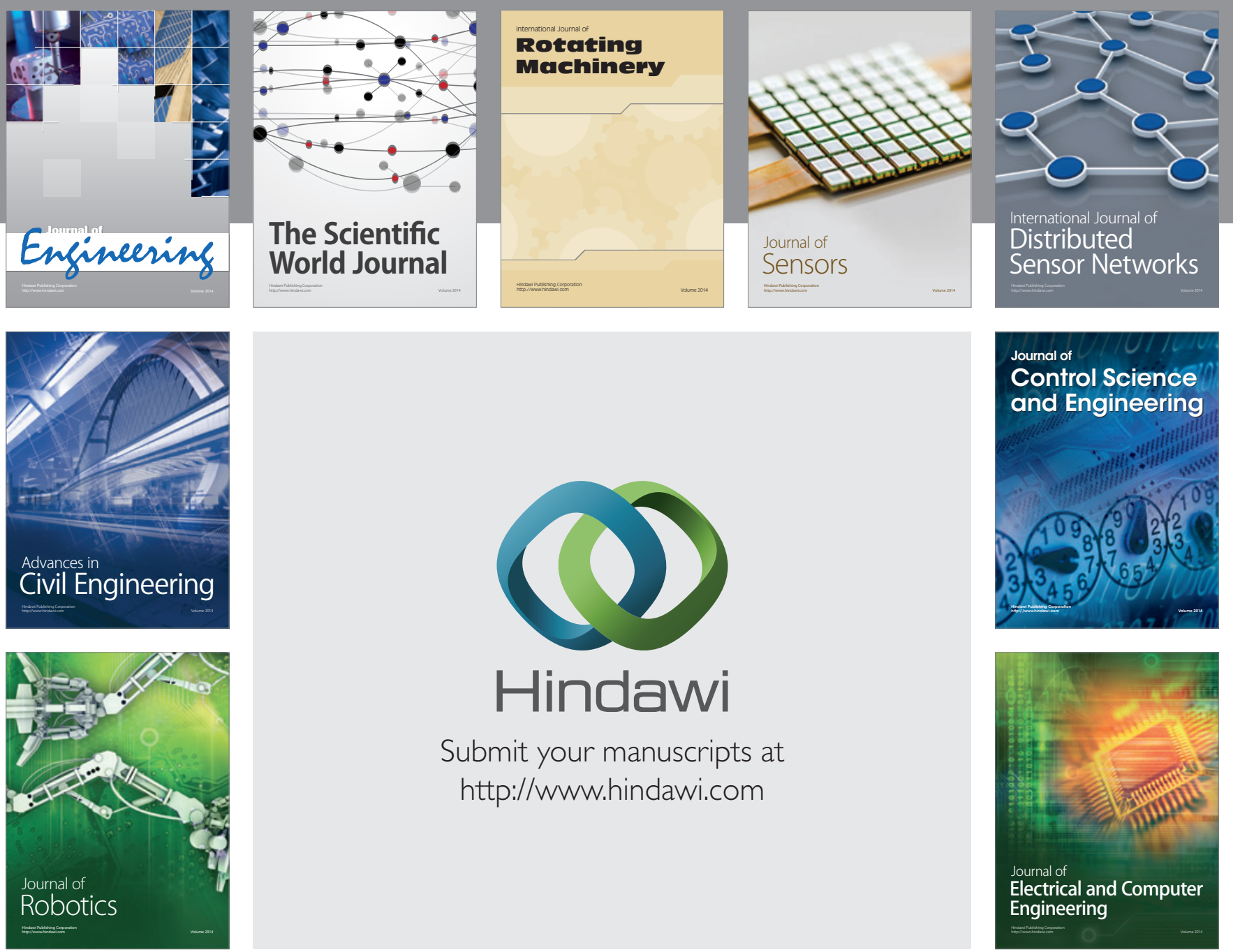

Submit your manuscripts at

http://www.hindawi.com
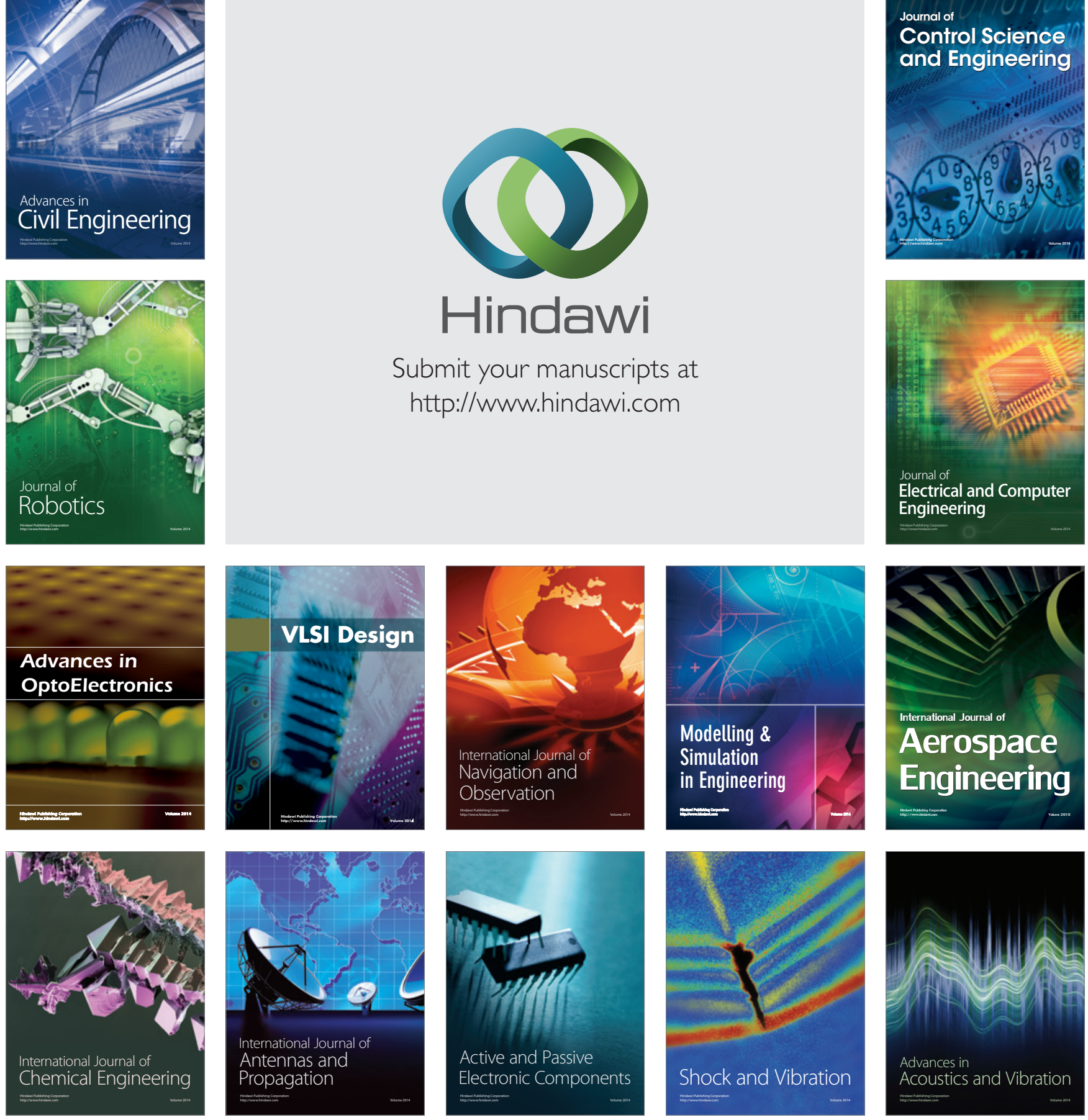